\title{
Gray-World Assumption on Perceptual Color Spaces
}

\author{
Jonathan Cepeda-Negrete and Raul E. Sanchez-Yanez \\ Universidad de Guanajuato DICIS \\ Salamanca, Guanajuato, Mexico \\ jonathancn@laviria.org, sanchezy@ugto.mx
}

\begin{abstract}
In this paper, the estimation of the illuminant in color constancy issues is analysed in two perceptual color spaces, and a variation of a well-known methodology is presented. Such approach is based on the Gray-World assumption, here particularly applied on the chromatic components in the CIELAB and CIELUV color spaces. A comparison is made between the outcomes on imagery for each color model considered. Reference images from the Gray-Ball dataset are considered for experimental tests. The performance of the approach is evaluated with the angular error, a metric well accepted in this field. The experimental results show that operating on perceptual color spaces improves the illuminant estimation, in comparison with the results obtained using the standard approach in RGB.
\end{abstract}

Keywords: Gray-World algorithm, color constancy, CIELAB, CIELUV.

\section{Introduction}

Color is an important feature for pattern recognition and computer vision fields. Typical applications include feature extraction [1], image classification [2, object recognition [34], scene categorization [56], human-computer interaction [7] and color appearance models [8]. Colors observed in images are determined by the intrinsic properties of objects and surfaces, as well as by the color of the illuminant. For a robust color-based system, effects generated by the illumination should be removed [9].

The ability of a system to recognize the correct colors, independently of the color source present in a scene, is known as color constancy [10. Remarkably, the human visual system has a natural capability to correct the color effects of the light source. However, the factors that are involved in this capability is not yet fully understood. The same process is not trivial to machine vision systems in an unconstrained scene [1].

Most color constancy algorithms use assumptions to simplify the computational estimation of the illuminant. The Gray-World (GW) 12 is one of the well-known approaches. This assumes that the average illumination reflected by the objects (reflectance) in a scene under a white light source, is achromatic. 
White Patch (WP) is another algorithm commonly used. It is based on the assumption that the maximum response in the channels of the RGB space is caused by a perfect reflectance [13. Some other methods rely on simple statistics of the images, like the Shades-of-Gray algorithm [14, the Gray-Edges (GE) algorithm [15] and the Local Color Space Average [16].

Originally the GW approach was proposed on the non-perceptual RGB color space, despite that color is a property of the human vision that depends on individual perception. Actually, most color constancy algorithms have been proposed and implemented in the RGB color space [17, and, in spite of the existence of a considerable number of methods, there is not a general solution for the color constancy problem.

Among the few research works addressing on the estimation of the illuminant on perceptual color spaces we can mention the study by Kloss [18, where the illuminant was estimated using WP and GW algorithms on CIELAB. Even though this method succeeds for chromatic adaptation purposes, the performance of the approach is not evaluated using the computed illuminant, leaving the accuracy of the estimation unverified. Similarly, experiments on the WP were done for image enhancement issues [19].

In this study, we propose the estimation of the illuminant directly on a perceptual color space. Thus, we focus on solving the color constancy problem. The GW assumption is analysed in two perceptual color spaces. Specifically, the well-known CIE $1976 L^{*} a^{*} b^{*}$ (CIELAB) and the CIE $1976 L^{*} u^{*} v^{*}$ (CIELUV) in order to provide a simple and fast transformation. For these two spaces, the Euclidean distance between two points in the space is proportionally uniform to the perceptual difference of the corresponding colors at the points [8]. The performance of the method is evaluated computing the accuracy between the estimated illuminant and a known reference value. Also, the outcomes using the standard GW approach on the non-perceptual RGB color space, are included for reference purposes. In addition, the GE algorithm is included in the comparison because of its outstanding performance 15 .

The rest of this paper is organized as follows. In Section 2, the GW algorithm, the color space transformations and our approach, are presented. Section 3 includes the experimental results in the test series, and the observations from the data obtained. Finally, the concluding remarks are given in Section 4.

\section{Methodology}

In this paper, the outcomes of GW approach in two perceptual color spaces are compared with those obtained using the standard GW, as depicted in Figure 1 .

Basically, the methodology consists in the next modules:

- Illuminant estimation using the Gray-World algorithm, for all images in RGB.

- Illuminant estimation using the Gray-World algorithm proposed, for all images in CIELAB. 


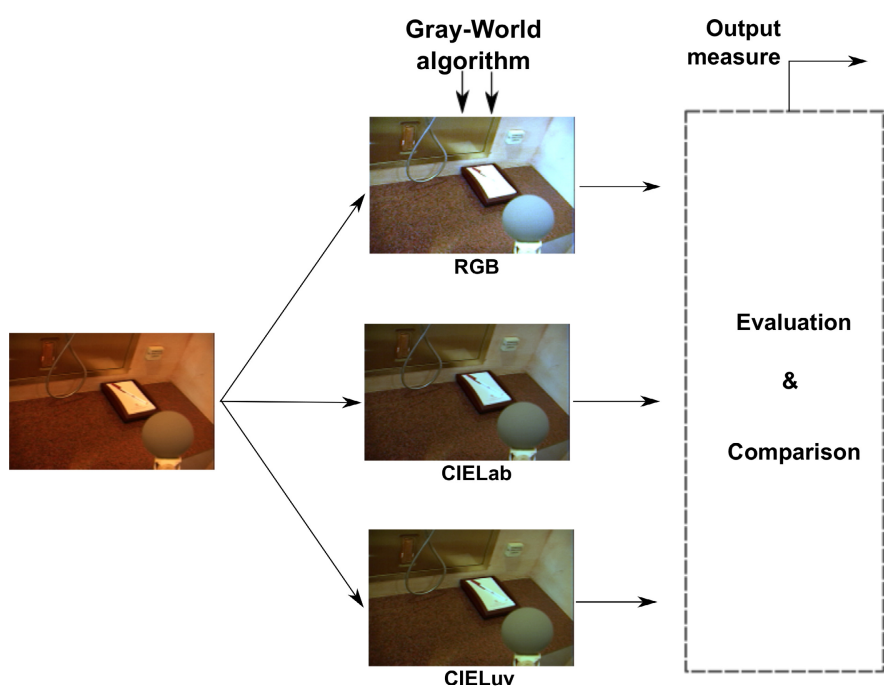

Fig. 1. Methodology for the experimental tests

- Illuminant estimation using the Gray-World algorithm proposed, for all images in CIELUV.

- The evaluation of the accuracy by the angular error between the illuminant estimated and its corresponding ground truth, for each approach.

- The comparison of the outcomes and the measures obtained.

The implementation of these modules requires the study of the image transformation from RGB to the CIELAB or CIELUV, and the corresponding inverse. Also, the assumption particular to the GW method must be given. These issues are addressed now.

\subsection{Image Transformation}

The algorithm considered throughout this work assumes that the illumination is uniform across the scene. Equation (1) gives the relationship for the color intensity,

$$
f_{i}(x, y)=G(x, y) R_{i}(x, y) I_{i}
$$

where, $f_{i}(x, y)$ is the pixel intensity at the position $(x, y)$ in an image or a frame, $G(x, y)$ is a factor that depends on the scene geometry, $R_{i}(x, y)$ is the reflectance of the object point displayed at position $(x, y)$ in the image and, $I_{i}$ is the illuminant in the scene. Index $i$ denotes the corresponding color channel in the image.

Once a color constancy algorithm is applied over an image $f_{i}(x, y)$, the outcome, $o_{i}(x, y)$, only depends on $G(x, y)$ and $R_{i}(x, y)$ assuming that $I_{i}=1$, that is, there is a white source illuminating the scene. In this case, 


$$
o_{i}(x, y)=\frac{f_{i}(x, y)}{I_{i}}=G(x, y) R_{i}(x, y)
$$

\subsection{Gray-World Assumption}

The GW assumption is the most popular algorithm for color constancy. Proposed by Buchsbaum [12, it is used as reference for other algorithms. The GW is based on the assumption that, on average, the real world tends to gray, and estimates the illuminant using the average color of all pixels. It is assumed that the information given by the average of each channel of the image is representative of the gray level.

The first step in the GW algorithm, is to compute the average $a_{i}$, as indicated in (3),

$$
a_{i}=\frac{1}{M N} \sum_{x=0}^{M-1} \sum_{y=0}^{N-1}\left\{f_{i}(x, y)\right\},
$$

where $M$ and $N$ are the number of columns and rows, respectively. Likewise, $a_{i}$ can be represented by

$$
\begin{gathered}
a_{i}=\frac{1}{M N} \sum_{x=0}^{M-1} \sum_{y=0}^{N-1} G(x, y) R_{i}(x, y) I_{i}, \\
a_{i}=I_{i} \frac{1}{M N} \sum_{x=0}^{M-1} \sum_{y=0}^{N-1} G(x, y) R_{i}(x, y), \\
a_{i} \approx I_{i} E\left[G R_{i}\right]=I_{i} E[G] E\left[R_{i}\right] .
\end{gathered}
$$

The function $E\left[G R_{i}\right]$ is the expected value of the geometry factor $G$ multiplied by the reflectance $R_{i}$. Both can be considered as independent random variables, as there is no correlation between the color and the shape of an object. Assuming that many different colors are present in the scene and each color is equally likely, the reflectance can be considered to be a random variable drawn from the range $[0,1]$, and

$$
\begin{gathered}
E[G] E\left[R_{i}\right]=E[G]\left(\int_{0}^{1} x d x\right)=E[G] \frac{1}{2}, \\
a_{i} \approx I_{i} E[G] \frac{1}{2}, \\
I_{i} \approx \frac{2}{E[G]} a_{i} .
\end{gathered}
$$

Once this global value is known, the illuminant $I_{i}$ is computed. Assuming that there is a perpendicular orientation between the object and the camera, $E[G]=1$.

$$
I_{i} \approx 2 a_{i}
$$

Since $f_{i}(x, y)=G(x, y) R_{i}(x, y) I_{i}$, the outcome image is given by

$$
o_{i}(x, y)=\frac{f_{i}(x, y)}{I_{i}}=\frac{f_{i}(x, y)}{2 a_{i}} .
$$




\subsection{Color Transformations}

The perceptual space transformations used in this study are applied to the CIEXYZ color space [20]. In order to transform an image from RGB to CIEXYZ, the RGB space needs to be determined. Here, sRGB is used because it is based in a colorimetric RGB calibrated space 21. All images need to be transformed from sRGB to CIEXYZ, applying (12) where $\{r, g, b\} \in[0,1]$ are the normalized color components

$$
\left[\begin{array}{l}
X \\
Y \\
Z
\end{array}\right]=\left[\begin{array}{lll}
0.4124 & 0.3576 & 0.1805 \\
0.2126 & 0.7152 & 0.0722 \\
0.0193 & 0.1192 & 0.9505
\end{array}\right]\left[\begin{array}{l}
r \\
g \\
b
\end{array}\right],
$$

whereas the inverse transformation is given by (13),

$$
\left[\begin{array}{l}
r \\
g \\
b
\end{array}\right]=\left[\begin{array}{rrr}
3.2410 & -1.5374 & -0.4986 \\
-0.9692 & 1.8760 & 0.0416 \\
0.0556 & -0.2040 & 1.0570
\end{array}\right]\left[\begin{array}{l}
X \\
Y \\
Z
\end{array}\right] .
$$

The color space CIELAB is computed from CIEXYZ using (14)-(17), obtaining the components of this space.

$$
\begin{aligned}
& L^{*}=116 f\left(Y / Y_{n}\right)-16, \\
& a^{*}=500\left[f\left(X / X_{n}\right)-f\left(Y / Y_{n}\right)\right], \\
& b^{*}=200\left[f\left(Y / Y_{n}\right)-f\left(Z / Z_{n}\right)\right], \\
& f(t)= \begin{cases}t^{1 / 3} & \text { if } t>\sigma^{3} \\
t /\left(3 \sigma^{2}\right)+16 / 116 & \text { otherwise, }\end{cases}
\end{aligned}
$$

where $X_{n}, Y_{n}$ and $Z_{n}$ are the coordinates of the reference white for the scene in CIEXYZ, $t$ can be $X / X_{n}, Y / Y_{n}$ or $Z / Z_{n}$, and $\sigma=6 / 29$.

For the inverse transformation, three intermediate variables are required, $f_{Y}$, $f_{X}$ and $f_{Z}$, shown in (18)-(20),

$$
\begin{gathered}
f_{Y}=\left(L^{*}+16\right) / 166, \\
f_{X}=f_{Y}+\left(a^{*} / 500\right), \\
f_{Z}=f_{Y}-\left(b^{*} / 200\right) .
\end{gathered}
$$

Finally, (21)-(23) are used to obtain the inverse transformation.

$$
\begin{aligned}
& Y= \begin{cases}Y_{n} f_{Y}^{3} & \text { if } f_{Y}>\sigma \\
f_{Y}-16 / 116 & \text { otherwise }\end{cases} \\
& X= \begin{cases}X_{n} f_{X}^{3} & \text { if } f_{X}>\sigma \\
f_{X}-16 / 116 & \text { otherwise }\end{cases} \\
& Z= \begin{cases}Z_{n} f_{Z}^{3} & \text { if } f_{Z}>\sigma \\
f_{Z}-16 / 116 & \text { otherwise }\end{cases}
\end{aligned}
$$


The transformation from XYZ to CIELUV begins by computing two intermediate variables, $u^{\prime}$ and $v^{\prime}$, given by

$$
\begin{aligned}
& u^{\prime}=\frac{4 X}{X+15 Y+3 Z}, \\
& v^{\prime}=\frac{9 Y}{X+15 Y+3 Z} .
\end{aligned}
$$

Also, it is necessary to obtain the variables $u_{n}^{\prime}$ and $v_{n}^{\prime}$, which are given by the coordinates for the reference white $\left(X_{n}, Y_{n}\right.$ and $\left.Z_{n}\right)$, using the same set of equations. Finally, the $L^{*}, u^{*}$ and $v^{*}$ components are computed applying the following equations

$$
\begin{gathered}
L^{*}=\left\{\begin{array}{cl}
(2 / \sigma)^{3} Y / Y_{n} & \text { if } Y / Y_{n} \leq \sigma^{3} \\
116\left(Y / Y_{n}\right)^{3}-16 & \text { otherwise, }
\end{array}\right. \\
u^{*}=13 L^{*}\left(u^{\prime}-u_{n}^{\prime}\right), \\
v^{*}=13 L^{*}\left(v^{\prime}-v_{n}^{\prime}\right),
\end{gathered}
$$

where $\sigma=6 / 29$. The inverse transformation is given by Equations (29)-(33).

$$
\begin{gathered}
u^{\prime}=\frac{u^{*}}{13 L^{*}}+u_{n}^{\prime} \\
v^{\prime}=\frac{v^{*}}{13 L^{*}}+v_{n}^{\prime} \\
Y=\left\{\begin{array}{c}
Y_{n} L^{*}(\sigma / 2)^{3} \quad \text { if } L^{*} \leq 8 \\
Y_{n}\left(\frac{L^{*}+16}{116}\right)^{3} \quad \text { otherwise }
\end{array}\right. \\
X=Y\left(\frac{9 u^{\prime}}{4 v^{\prime}}\right) \\
Z=Y\left(\frac{12-3 u^{\prime}-20 v^{\prime}}{4 v^{\prime}}\right)
\end{gathered}
$$

\subsection{Gray-World Assumption on Perceptual Color Spaces}

The Gray-World theory assumes that the average color in an image tends to a gray color. In other words, the mean of each chromatic component tends to this gray color. This assumption, originally was applied to the RGB color space, which has three color components. We propose to apply this assumption on two perceptual color spaces.

The reason for transforming the input image into a perceptual color space is the estimation of the illuminant. Perceptual color spaces are conformed by two chromatic components, additionally to lightness. These two chromatic components are used in our approach while the lighting information is omitted. 
CIELAB is the first perceptual color space taking into account. This space is perceptually uniform, and the chromatic components, $a^{*}$ and $b^{*}$, are not correlated with the lightness component $L^{*}$. This last, corresponding to a perceptual scale of brightness and excluding any color information. Given an image $f_{i}(x, y)$, where $i \in\{R, G, B\}$, we compute the component of each pixel, $L^{*}, a^{*}$, and $b^{*}$, of the corresponding image on the CIELAB space, using Eqs. (12) and (14)-(17). Following the Gray-World assumption, the average value for each chromatic component is then computed as

$$
\begin{aligned}
& I_{a^{*}}=\frac{1}{M N} \sum_{y=0}^{M-1} \sum_{x=0}^{N-1} a^{*}(x, y), \\
& I_{b^{*}}=\frac{1}{M N} \sum_{y=0}^{M-1} \sum_{x=0}^{N-1} b^{*}(x, y),
\end{aligned}
$$

where $M$ and $N$ are the number of columns and rows in the image, respectively. These two values are very important because they represent the color of the illuminant in this perceptual space.

In order to increase the lighting in the outcome image, for completing the illuminant estimation we suggest the use of $I_{L^{*}}=\max \left\{L^{*}(x, y)\right\}$ as a third component. That is, we assume that the lightness value for the approximate gray is the highest possible in the image. Experimental tests support this assumption. Therefore, we can consider this approach as a combination between the WhitePatch and the Gray-World methods.

Now, the output image will be that obtained using the calculated illuminant. For this purpose, such illuminant must be in the RGB space. For the computation of $I_{i \in\{R, G, B\}}$, the $I_{j \in\left\{L^{*}, a^{*}, b^{*}\right\}}$ illuminant is mapped by the sequential inverse transformations from CIELAB to XYZ using (18)-(23), and then from XYZ to RGB using Eq. (13). Finally, the accuracy for the illuminant $I_{i}$ is evaluated and the outcome image is computed using (2).

CIELUV is the second perceptual color space considered in this study. Although it has some similar properties than CIELAB (two chromatic components and lightness), CIELUV incorporates a different formulation for the chromatic adaptation. Transformations in this space are performed using Equations (24)(33), applied in a manner similar to the aforementioned process.

\section{Experimental Results}

In order to test our approaches, we compare their performance with the corresponding outcomes using the standard approach in RGB. We use the Gray-Ball 22. benchmark image set for the experiments. This image set is commonly used for the evaluation of color constancy algorithms as it is labeled with ground truth illuminants. More than 11000 images belong to this image set, however most of these images are highly correlated. Thus, Bianco et al. [5] propose the use of a subset containing approximately the $10 \%$ of the complete set. We use this subset, which includes 1135 images. 


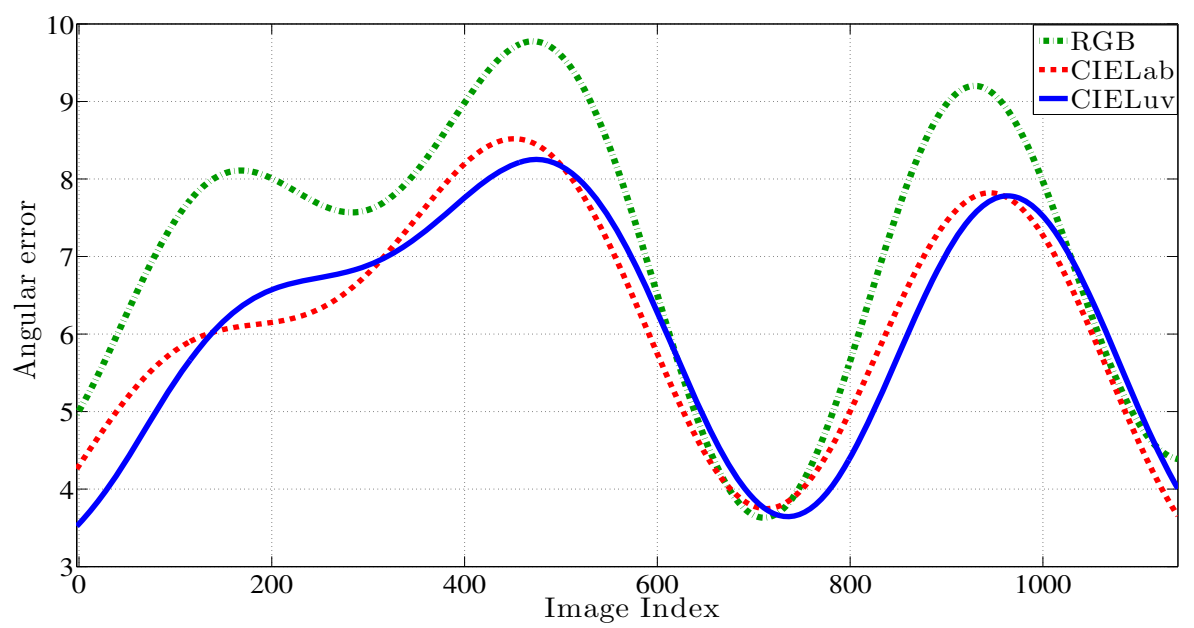

Fig. 2. Curve fitting for each approach showing the trend of the angular errors given by the outcomes. The lower the angular error is, the better the estimation is.

Table 1. Statistical angular errors for the different approaches

\begin{tabular}{|c|c|c|c|}
\hline Algorithm & Mean & Median & Max \\
\hline \hline Gray-World (RGB) & 7.2 & 6.0 & 33.3 \\
Gray-World (CIELAB) & 6.5 & 5.7 & 25.9 \\
Gray-World (CIELUV) & 6.2 & 5.5 & 27.1 \\
$2^{\text {nd }}$ order Gray-Edge & 6.1 & 5.1 & 24.0 \\
$1^{\text {st }}$ order Gray-Edge & 6.0 & 4.9 & 23.7 \\
\hline
\end{tabular}

\subsection{Performance Evaluation}

In order to evaluate the performance of the color constancy algorithms, a metric of performance must be considered. In this case, color constancy must be evaluated according to the estimated color of the illuminant. Hordley and Finlayson 23. proposed a metric well suited for the evaluation of the color constancy, the angular error. In such study, they discussed three good descriptors for the angular error distribution, which are the mean, median and maximum error values. This metric is given by

$$
\mathrm{e}_{\text {ang }}=\cos ^{-1}\left(\frac{\mathbf{I}_{r} \mathbf{I}_{e}}{\left|\mathbf{I}_{r}\right|\left|\mathbf{I}_{e}\right|}\right),
$$

where $\mathbf{I}_{r}$ is the known illuminant in the scene (ground truth) and $\mathbf{I}_{e}$ is the illuminant estimated by an algorithm under test. It is important to note that for the evaluation of the color constancy, a ground truth is always necessary. These reference values should be provided by the authors of the image dataset. We must note that the lower the angular error is, the better the estimation is. 

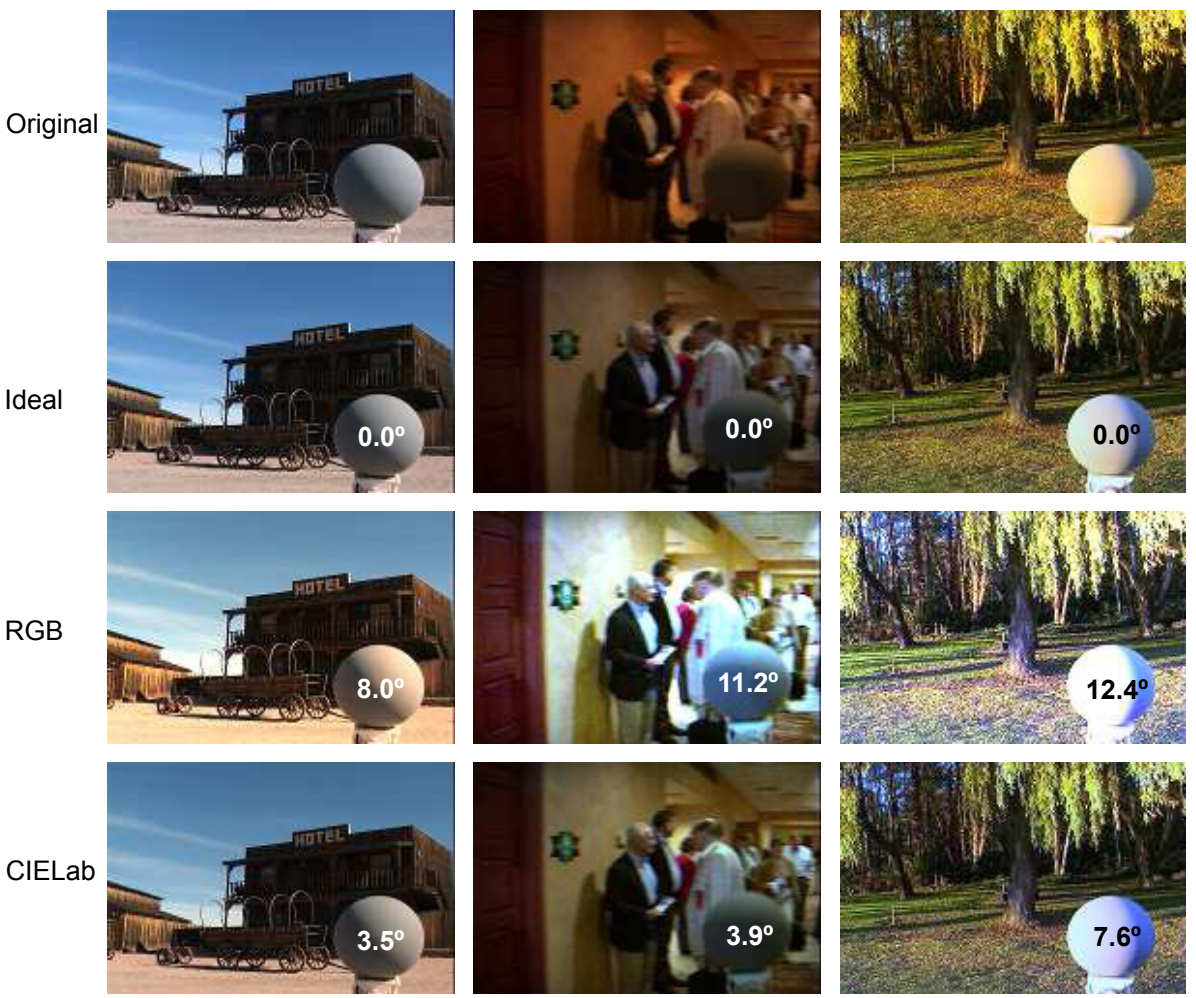

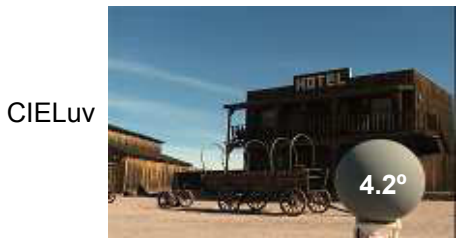

a)

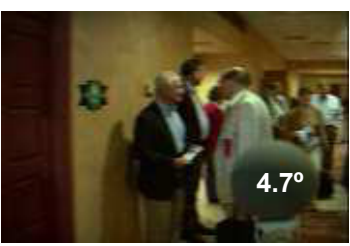

b)

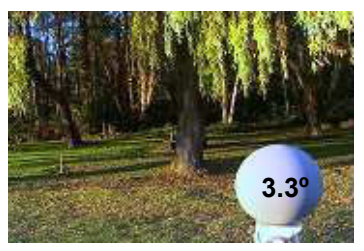

c)

Fig. 3. Three examples out of the 1135 images where the angular error is shown in the gray ball. The ideal image is included. a) ApacheTrial frame no. 01520, b) CIC2002 frame no. 10150, c) DeerLake frame no. 00140.

Table 2. Computing time for each approach

\begin{tabular}{|c|r|}
\hline Algorithm & Time (ms) \\
\hline \hline Gray-World (RGB) & 0.57 \\
Gray-World (CIELUV) & 15.73 \\
Gray-World (CIELAB) & 31.22 \\
$1^{\text {st }}$ Gray-Edge & 130.44 \\
$2^{\text {nd }}$ Gray-Edge & 216.20 \\
\hline
\end{tabular}




\subsection{Data Obtained and Comparison}

The experiment was conducted on a comparative analysis between the outcomes from our approaches and the outcomes from the classical approach using RGB discussed in Section 2. Therefore, 1135 illuminants were obtained, and the angular error was calculated for these outcomes. In order to clarify the behavior of the outcomes, the angular errors obtained were approximated by curve fitting. This approximation was computed using three terms of the Fourier Series, such that the fitted curve describes the trend of the particular approach, as shown in Fig. 2,

Three examples of outcomes using the algorithms under evaluation are shown in Fig. 3. Each image is presented under diverse light conditions and is processed using the three approaches. In addition, the corresponding ideal image is shown. An ideal image is one that is corrected using the ground truth illuminant. GW applied in RGB, generally introduces a saturation which is not found in the source images. In contrast, this algorithm applied on perceptual color spaces preserves better the relation of the colors. We can appreciate this in Fig. 3 .

Table 1shows the three descriptors for the angular error distribution suggested in Section 4.1. According to these descriptors, the application of GW on any perceptual color space is significantly better than using RGB. However, the GW algorithm applied on CIELUV is marginally better than the algorithm on CIELAB. The Gray-Edge (GE) algorithm is included in the experiments for comparison purposes. This algorithm has shown the best performance for this image set [5], and belongs to the group of static color constancy algorithms. However, this has the particular disadvantage of requiring a tuning process using training images. Particularly, the method depends of three parameters, which must be correctly chosen to improve the performance. For this work, GE operates using the parameters proposed by Bianco et al. 24]. Nevertheless, the difference in performance between GW using a perceptual space and GE is very small. The main difference is that the GW algorithm in any color space does not require any tuning process.

Table 2 shows the processing time taken by each approach. The specifications of the computer are the following: iMac Apple, $2.5 \mathrm{GHz}$ Intel Core i5, 4GB ram $1333 \mathrm{MHz}$ DDR3. We can appreciate that the difference in computing time between GE and GWs approaches is significant. The GW algorithm using CIELUV was the best in performance, except in comparison with GE. However, GW is about 8.3 times faster than the $1^{\text {st }}$ order GE, and 13.7 times faster than the $2^{\text {nd }}$ order GE.

\section{Conclusion}

A variation of the method using the GW assumption for color constancy has been analyzed in the CIELAB and CIELUV color spaces. Given a test image, the illuminant is estimated and then compared against a reference value. Experimental tests are conducted comparing the outcomes of the proposed methods with outcomes using the standard GW in RGB and the GE algorithm. According to the 
results, we conclude that outcomes from our approach, a GW assumption in a perceptual color space, are better than those obtained using the standard procedure in RGB. Despite that the outcomes using the GE algorithm are slightly better than those using our approach, for practical applications we can choose the latter, because it is significantly faster and does not require a tuning process. Also, we can appreciate that GW on CIELUV is marginally better than on CIELAB according to the accuracy of the estimated illuminant. Moreover, the processing time is considerably faster on CIELUV. For these reasons, our approaches, particularly the GW using CIELUV, can be considered well-suited preprocessing methods for real-time applications in the pattern recognition and computer vision fields.

Acknowledgments. Jonathan Cepeda-Negrete thanks to the Mexican National Council on Science and Technology, CONACyT, for the financial support provided via the scholarship 290747 (Grant No. 388681/254884), to the University of Guanajuato for the financial support provided via the PIFI-2012 program and the DAIP FJI scholarship.

\section{References}

1. Gevers, T., Smeulders, A.W.M.: PicToSeek: Combining Color and Shape Invariant Features for Image Retrieval. IEEE Trans. Image Process. 9(1), 102-119 (2000)

2. Schroeder, M., Moser, S.: Automatic Color Correction Based on Generic ContentBased Image Analysis. In: Proc. of Color Imaging Conference, pp. 41-45 (2001)

3. Gasparini, F., Schettini, R.: Color balancing of digital photos using simple image statistics. Pattern Recognition 37(6), 1201-1217 (2004)

4. van de Weijer, J., Schmid, C., Verbeek, J.: Using High-Level Visual Information for Color Constancy. In: Proc. of the Inter. Conf. on Computer Vision, pp. 1-8 (2007)

5. Bianco, S., Ciocca, G., Cusano, C., Schettini, R.: Improving Color Constancy Using Indoor-Outdoor Image Classification. IEEE Trans. Image Process. 17(12), 2381$2392(2008)$

6. Gijsenij, A., Gevers, T.: Color Constancy using Natural Image Statistics. In: IEEE Conf. on Computer Vision and Pattern Recogn., pp. 1-8 (2007)

7. Yang, J., Stiefelhagen, R., Meier, U., Waibel, A.: Visual tracking for multimodal human computer interaction. In: Proc. of the Conference on Human Factors in Computing Systems, pp. 140-147 (1998)

8. Fairchild, M.D.: Color Appearance Models, 2nd edn. John Wiley \& Sons (2005)

9. Gijsenij, A., Gevers, T., van de Weijer, J.: Computational Color Constancy: Survey and Experiments. IEEE Trans. Image Process. 20(9), 2475-2489 (2011)

10. Zeki, S.: A vision of the brain. John Wiley \& Sons (1993)

11. Agarwal, V., Abidi, B.R., Koshan, A., Abidi, M.A.: An Overview of Color Constancy Algorithms. J. Pattern Recogn. Res. 1, 42-54 (2006)

12. Buchsbaum, G.: A spatial processor model for object colour perception. Journal of the Franklin Institute 310, 1-26 (1980)

13. Land, E.H., McCann, J.J.: Lightness and Retinex Theory. J. Opt. Soc. Am. 61(1), $1-11(1971)$ 
14. Finlayson, G.D., Trezzi, E.: Shades of Gray and Colour Constancy. In: Proc. of Color Imaging Conf., pp. 37-41 (2004)

15. van de Weijer, J., Gevers, T., Gijsenij, A.: Edge-Based Color Constancy. IEEE Trans. Image Process. 16(9), 2207-2214 (2007)

16. Ebner, M.: Color constancy based on local space average color. Machine Vision and Applications 20, 283-301 (2009)

17. Ebner, M.: Color Constancy. John Wiley \& Sons (2007)

18. Kloss, G.K.: Colour Constancy using von Kries Transformations Colour Constancy goes to the Lab. Res. Lett. Inf. Math. Sci. 13, 19-33 (2009)

19. Cepeda-Negrete, J., Sanchez-Yanez, R.E.: Experiments on the White Patch Retinex in RGB and CIELAB color spaces. Acta Universitaria 22(NE-1), 21-26 (2012)

20. Colorimetry, S.J.: Understanding the CIE System. John Wiley \& Sons (2007)

21. Stokes, M., Anderson, M., Chandrasekar, S., Motta, R.: A Standard Default Color Space for the Internet - sRGB. Hewlett-Packard, Microsoft (1996)

22. Ciurea, F., Funt, B.: A Large Image Database for Color Constancy Research. In: Proc. of the IS\&T/SID Eleventh Color Imaging Conf., pp. 160-164 (2003)

23. Hordley, S.D., Finlayson, G.A.: A Re-evaluation of Colour Constancy Algorithms. In: Proc. of 17th Inter. Conf. on Pattern Recog (ICPR), pp. 76-79 (2004)

24. Bianco, S., Ciocca, G., Cusano, C., Schettini, R.: Automatic color constancy algorithm selection and combination. Pattern Recogn. 43(3), 695-705 (2010) 